Apidologie, 1973, 4 (4), 331-339.

\title{
EIN BEITRAG ZUR LABORDIAGNOSE DER BÖSARTIGEN FAULBRUT DER HONIGBIENE UNTER BESONDERER BERÜCKSICHTIGUNG DER IMMUNOFLUORESZENZMETHODE
}

\author{
Contribution au diagnostic de la loque américaine des abeilles \\ par une méthode d'immunofluorescence
}

Elmar OTTE

Institut für Fisch-und Bienenliunde der Tierärztlichen

Hochschule in Wien 1030 Wien

\section{SUMMARY}

\section{CONTRIBUtion TO THE LABORATORY DIAGNOSIS OF AMERICAN FOULBROOD (A.F.B.) OF THE HONEYBEE WITH PARTICULAR REFERENCE TO THE FLUORESCENT ANTIBODY TECHNIQUE}

The different laboratory methods available for the diagnosis of A.F.B. are reviewed. In general, no laboratory examination is necessary for the experienced bee-keeper to recognize the advanced stage of the disease, but on the other hand a number of problems may arise which can be satisfactorily solved with the help of laboratory investigations only. Among these problems are the detection of the disease in the initial stage or of cryptogenic infections; the demonstration of vegetative forms of $B$. larvae, examination of honey for A.F.B. spores, detection of A.F.B. spores in waste accumulated during the winter, etc.

With the introduction of the fluorescent antibody technique the diagnostic possibilities so far available have not only been expanded, but improved to such an extent, that now it has become possible to visualize a few spores in sediments or other samples submitted for examination. Previously, when only a small number of spores was present in the infected material, cultivation of $B$. larvae was necessary to diagnose A.F.B. Unfortunately, the honey and other materials are largely contaminated with various banal sporulating germs which are much more resistant to heat and other environmental factors than are the spores of $B$. larvae. In the cultures the colonies formed by these organisms generally grow more vigorously and more rapidly than the colonies of $B$. larvae, and this may lead to such an overgrowth of the former that an accurate diagnosis cannot be established. 
These sources of error have been eliminated with the use of the fluorescent antibody technique, but in this case it is very difficult to fix the smears so as to revent them from being washed off the slide during the repeated washings required. Marked cross reactions with related spore-bearing organisms were not observed. 36 strains of bacteria were tested. $B$, anthracis gave a concurrent reaction. It is still obscure whether certain banal sporulating organisms may give pronounced concurrent reactions, but this appears rather unlikely. Since definite antigenic differences exist between spores and vegetative forms of $B$. larvae, it is necessary to use varying conjugates according to the case.

\section{ZUSAMMENFASSUNG}

Es werden die einzelnen gebräuchlichen Labormethoden zur Erkennung der bösartigen Faulbrut besprochen. Während in der Regel der erfahrene Bienenzüchter zur Erkennung der fortgeschrittenen Krankheit keine Laboruntersuchung braucht, ergeben sich eine Reihe von Fragestellungen, die man nur durch eine Laboruntersuchung klären kann : die Erkennung von beginnenden oder kryptogenen Infektionen, der Nachweis der Vermehrungsformen des B. larvae, Honiguntersuchungen auf Faulbrutsporen, Nachweis der Faulbrutsporen im Wintergemüll usw.

Die bisher angewandten diagnostischen Möglichkeiten werden durch die Neueinführung der Immuno fluoreszenz in die Diagnostik erweitert und so verfeinert, daß es sogar gelingt, nur wenige Sporen in einem Sediment oder einer Probe spezifisch sichtbar zu machen. Bisher war der Nachweis weniger Sporen nur durch den Kulturversuch möglich. Leider gibt es aber im Honig oder in anderem Untersuchungsmaterial auch noch andere banale Sporenbildner, die gegen Temperatur u. a. Faktoren wesentlich resistenter als Faulbrutsporen sind. Die Kolonien dieser banalen Sporenbildner wachsen auf dem Nährboden viel kräftiger und rascher als $B$. larvae und verhindern durch das Überwuchern der B. larvae-Kolonien häufig eine eindeutige Diagnose.

Bei der Immunofluoreszenz fallen diese Fehlerquellen weg, andererseits ist es aber sehr schwierig, die Ausstriche, die mehrmals gut gewaschen werden müssen, am Objektträger festzuhalten. Starke Kreuzreaktionen mit verwandten Sporenbildnern konnten nicht festgestellt werden. Es wurden 36 Bakterienstämme überprüft. Bei Milzbrandbakterien wurde eine Mitreaktion nachgewiesen. Die Möglichkeit, daß es banale Sporenbildnerarten gibt, die stärker mitreagieren könnten, bleibt noch offen, ist aber unwahrscheinlich. Da aber eindeutige antigene Unterschiede zwischen Sporen und vegetativen Bakterienformen bei $B$. larvae bestehen, ist es erforderlich, je nach Fragestellung ent prechende Konjugate zu verwenden.

\section{EINLEITUNG}

Die bösartige Faulbrut wurde bis vor kurzem als Krankheit der älteren Larvenstadien der Honigbiene angesehen, daher der Name Nymphenseuche. Seit den Untersuchungen von Schulz-langner (1956) wird angenommen, daß auch regelmäßig die frühen Entwicklungsstadien der Larven befallen werden. Epidemiologisch sind die verdeckelten erkrankten und abgestorbenen Larven von besonderer Bedeutung. Während die erkrankten Larven der unverdeckelten Brut von einem kräftigen Volke rasch beseitigt werden, bleiben die verdeckelten abgestorbenen Larven lange Zeit in den Waben erhalten, wobei es zur Versporung und Konservierung der Bakterien kommt. Die Krankheit kann dann durch die sehr widerstandsfähigen Sporen relativ leicht verbreitet werden, überhaupt dann, wenn die betroffenen Völker einen verminderten Putztrieb besitzen und die Imker die Hygiene am Bienenstand außer acht lassen. 
Die Diagnose der bösartigen Faulbrut bereitet bei Fällen, bei denen die Krankheit bereits die verdeckelte Brut befallen hat, kaum Schwierigkeiten. Jeder erfahrene Praktiker erkennt die fortgeschrittene Krankheit bereits auf Grund der pathologischen Veränderungen mit dem freien Auge. Verdachtsmomente ergeben sich aus lückenhafter Brut, verfärbten eingesunkenen oder durchlöcherten Zelldeckeln, graugelb bis dunkelbraun verfärbten, verflüssigten Nymphen. Pathognomonisch ist die stark fadenziehende schleimige Beschaffenheit der verflüssigten Larven (Zündholzprobe).

Es gibt auch Grenzfälle, besonders beim ersten Auftreten der Krankheit auf einem Bienenstand, bei denen die Symptome undeutlich hervortreten. Etwas schwieriger ist das Erkennen der Krankheit an der unverdeckelten Brut. Einen wichtigen, aber unsicheren Hinweis ergibt in solchen Fällen eine Gelbverfärbung der Junglarven. Erkrankte Junglarven verlieren ihre glänzende und gegliederte Oberfläche. Zur Identifizierung der Krankheit gibt es einige sehr brauchbare Labormethoden :

1) Nachweis der Geißelzöpfe mittels Geißeldarstellungen,

2) Darstellung der Geißelzöpfe im Phasenkontrastverfahren oder im Dunkelfeld,

3) Serologische Nachweismethoden,

4) Kulturversuch,

5) Anwendung der Immuno fluoreszenz.

Die ersten zwei Verfahren sind zum Nachweis einer bereits länger bestehenden Krankheit bei älteren Bienenlarven geeignet. Die serologischen Nachweismethoden, das Kulturverfahren und die Immunofluoreszenz eignen sich jedoch auch zum Nachweis einer erst beginnenden Krankheit bei Junglarven.

\section{BESPRECHUNG DER DIAGNOSTISCHEN METHODEN}

\section{1. — Nachweis der Geißelzöpfe mittels Geißeldarstellungen}

Im Verlaufe der Versporung des $B$. larvae entstehen ganz typische, für diese Bakterienart spezifische Geißelzöpfe, die an Spirillen erinnern. Es werden zu ihrer Darstellung verschiedene Verfahren angegeben. Am geeignetsten unter den zahlreichen beschriebenen Verfahren erscheint eine von Werner (1935) erprobte und hier laufend angewandte Methode :

Hiezu ist die Beize nach Gray erforderlich, die von uns etwas modifiziert wurde und sich wie folgt zusammensetzt :

5 Teile einer wässerigen Lösung von Chromkalialaun (1 Teil Chromkalialaun in 10 Teilen dest. Wasser)

2 Teile einer konzentrierten Sublimatlösung (1 Teil Sublimat in 16 Teilen dest. Wasser)

2 Teile einer $20 \%$ Tanninlösung $(2 \mathrm{~g}$ Tannin in $10 \mathrm{ml}$ dest. Wasser). 
Weiters benötigt man eine ZETrNow'sche Silberlösung, die nach folgendem Rezept hergestellt wird :

$5 \mathrm{~g}$ Silbernitrat und $6 \mathrm{~g}$ Natriumsulfat werden in $30 \mathrm{ml}$ dest. Wasser gelöst. Den entstehenden Niederschlag läßt man absetzen, die überstehende Flüssigkeit wird verworfen. Hierauf wird der Niederschlag $(2.3 \mathrm{mg})$ mit etwa $20 \mathrm{ml}$ destilliertem Wasser gewaschen und schließlich in $500 \mathrm{ml}$ destilliertem Wasser suspendiert, geschüttelt und der Rest des Niederschlages wieder absetzen gelassen. Die überstehende Flüssigkeit (Silbersulfatlösung )wird abgegossen und aufgehoben.

Die ZeTtrow'sche Silberlösung wird nun hergestellt, indem man $25 \mathrm{ml}$ Silbersulfatlösung mit $25 \mathrm{ml}$ destilliertem Wasser verdünnt und tropfenweise eine $33 \%$ Äthylaminlösung langsam zusetzt. Zuerst entsteht hiebei ein brauner trüber Niederschlag, der sich bei weiterer vorsichtiger Zugabe von Äthylamin wieder löst. Ganz wesentlich für das Gelingen der Geißelfärbung ist die genaue Einstellung der Silberlösung. Die Silberlösung ist nur dann richtig, wenn sich der braune Niederschlag eben erst gelöst hat. GrAY'sche Beize, Silbersulfatlösung und ZETTNOW'sche Lösung sind unbegrenzt haltbar.

Herstellung der Präparate : Auf einem ganz sauberen Deckglas oder einem gut gereinigten Objektträger werden ganz dünne Ausstriche von verdächtigem Untersuchungsmaterial (z. B. Larven oder Schorfe) hergestellt. Die luftgetrockneten Präparate werden 5-6 Minuten mit GRAY' scher Beize behandelt. Hierauf werden die Ausstriche gut mit Wasser abgewaschen und wieder getrocknet. Auf die trockenen Ausstriche kommt nun die Zetrnow'sche Silberlösung. Der Ausstrich wird anschließend so lange mit der Sparflamme eines Bunsenbrenners erwärmt, bis die Silberlösung leicht dampft und sich der Ausstrich bräunt und sich schließlich zu schwärzen beginnt. Nach dem Waschen und Trocknen des Ausstriches kann dieser sofort mit einem Immersionsobjektiv beurteilt werden. Die Geißelzöpfe, Bakterien und Sporen treten deutlich schwarz hervor.

\section{2. - Darstellung der Geißelzöpfe im Phasenkontrastverfahren oder im Dunkelfeld}

Ein Nachweis der Geißelzöpfe und Sporen im Phasenkontrastverfahren ist möglich. Die Ausbeute an sichtbaren Geißelzöpfen oder einzelnen Geißelelementen ist aber sehr gering. Dieses Verfahren gibt nur einen kleinen Bruchteil der tatsächlich vorhandenen Geißelelemente wieder. Das Phasenkontrastmikroskop ist daher kaum in der Lage, das Verfahren von Werner zu ersetzen. Etwas ergiebiger ist es, die Untersuchung mit dem Dunkelfeldmikroskop auszuführen. Hiebei werden die Geißelzöpfe und Sporen regelmäßiger und relativ deutlich dargestellt.

\section{3. - Serologischer Nachweis der bösartigen Faulbrut}

Von Krockow (1925) und Poltew (1958) wurde gezeigt, daß B. larvae bei Kaninchen Antikörperbildung auslösen kann. Es konnten sowohl agglutinierende, präzipitierende als auch komplementbindende Antikörper gegen $B$. larvae erzeugt werden. Poltew benützte diese Erfahrung, um vor allem 
mit spezifischen präzipitierenden Kaninchenseren eine serologische Nachweismethode zur Erkennung und Differenzierung von Brutkrankheiten zu erarbeiten. Bei diesem Verfahren werden die spezifischen diagnostischen Sera mit den Auszügen aus dem Untersuchungsmaterial (Zelleninhalt, Totenfall von Bienen usw.) in einem Röhrchen überschichtet, wobei es bei Anwesenheit von Faulbrutantigenen in der Grenzschicht zwischen Serum und Auszug zu einer typischen weißen Ringbildung kommt, ähnlich wie bei der Thermopräzipitation nach Ascolı beim Milzbrand. Interessant ist, daß bei $B$. larvae unterschiedliche somatische Geißel- und Sporenantigene festgestellt wurden. Dementsprechend muß auch je nach Fragestellung die Wahl der diagnostischen Sera erfolgen.

\section{4. - Darstellung der Faulbruterreger im Kulturversuch}

Ein sehr sicherer, allerdings etwas länger dauernder Weg ist es, im Kulturversuch den $B$. larvae darzustellen. Es werden in der Literatur eine Reihe von Nährmedien angegeben, auf denen der $B$. larvae wächst. In der Praxis hat sich zunächst ein Traubenzucker-Schafblutagar gut bewährt. In allen uns bisher zugänglichen Fällen von bösartiger Faulbrut gelang es auf diesem Medium zumeist, Reinkulturen von $B$. larvae zu isolieren. Nach längeren Versuchen habe ich einen Nährboden zusammengestellt, auf dem sich $B$. larvae besonders gut vermehrt :

$\begin{array}{lr}\text { Hefeextrakt BBL } & 10,0 \mathrm{~g} \\ \text { Laevulose } & 0,5 \mathrm{~g} \\ \text { Dextrose } & 0,5 \mathrm{~g} \\ \text { Lösliche Stärke } & 10,0 \mathrm{~g} \\ \text { Pepton trypt. oder pept. verdaut } & 1,0 \mathrm{~g} \\ \mathrm{KH}_{2} \mathrm{PO}_{4} & 2,0 \mathrm{~g} \\ \mathrm{Na}_{2} \mathrm{HPO}_{4} .2 \mathrm{H}_{2} \mathrm{O} & 1,0 \mathrm{~g} \\ \text { Agar } & 20,0 \mathrm{~g} \\ \text { Magermilchpulver } & 0,5 \mathrm{~g} \\ \text { Eipulver (Vollei) } & 0,5 \mathrm{~g} \\ \text { Aqua dest. } & 1000,0 \mathrm{ml}\end{array}$

Das angegebene Grundmedium wird bei 3 atü 15 Minuten sterilisiert und nach dem Abkühlen auf 50 Grad C im Wasserbad mit 50-100 ml sterilem defibriniertem Schafblut versetzt, gut geschüttelt und in sterilen Petrischalen ausgegossen.

Bisher gelang es damit, den Faulbruterreger regelmäßig in der vegetativen Phase zu züchten. Versuche, eine reichliche Versporung in der Kultur auszulösen, mißlangen. Es wurden spezielle Nährböden nach Angabe von Forster et al. (1950) zur Versporung hergestellt. Große Mengen von Kulturmaterial 
wurden in dest. Wasser, diversen Salzlösungen und bei verschiedenen pH Werten längere Zeit aufbewahrt. Es wurde ferner versucht, unter anaeroben oder mikroaerophilen Bedingungen die Versporung herbeizuführen. Die Kulturen wurden verschiedenen Temperaturen bis zu 100 Grad C ausgesetzt.

Die Kolonien des $B$. larvae sind charakteristisch mattgrau, opak, wachsen innerhalb von 48 Stunden bei 34 Grad C gut an. Sie besitzen in der Regel einen rauhen Rand, zum Teil mit kurzen zugespitzten Ausläufern ähnlich wie bei Milzbrandkulturen. Durch sein typisches Wachstum ist der B. larvae bereits ohne weitere Differenzierungsmethoden abgrenzbar. Im mikroskopischen Bild erscheint er in Form von sehr langen grampositiven Stäbchen. Vereinzelte Sporen finden sich in älteren Kulturen häufig.

\section{5. — Der Nachweis der Faulbrutsporen mit der Immunofluoreszenzmethode}

Bisher haben sich nur wenige Untersucher mit dem Problem des Nachweises von Faulbruterregern mit dieser Methode befasst. Erstmals wurde in den Arbeiten von Toschкov et al. (1969 u. 1970) berichtet, daß diese Methode sehr genaue Resultate zu liefern vermag. Leider fehlten diesen Arbeiten exakt reproduzierbare methodische Angaben über die Gewinnung wirklich brauchbarer Konjugate. Ich habe aus diesem Grunde zwei Kaninchengruppen immunisiert. Zuerst wurden in kurzen Abständen intravenös und intraperitoneal kleinere Antigenmengen verabreicht. Später erfolgte eine weitere Steigerung des Antikörpertiters durch subcutane Verabreichung von Antigen mit inkomplettem Freundschem Adjuvans in die Vorderpfoten. Beim Erreichen entsprechend hoher Serumtiter wurden die Kaninchen durch Herzpunktion ausgeblutet.

Die erste Gruppe Kaninchen wurde mit unversportem lebendem Kulturmaterial von $B$. larvae immunisiert. Die zweite Gruppe der Kaninchen wurde mit gewaschenen Sporenaufschwemmungen aus Faulbrutschorfen geimpft, da ich trotz langwieriger Versuche nicht in der Lage war, Faulbrutkulturen zur vollständigen Versporung zu bringen.

Verarbeitung der Sera : Die Kaninchenseren werden zuerst 1 : 5 mit einem 0,15 mol $\mathrm{PO}_{4} \mathrm{NaCl}-\mathrm{Puffer} \mathrm{pH} \mathrm{7,2}$ verdünnt und im Verhältnis von 5 Teilen verdünntem Serum zu 4 Teilen gesättigter (50\%) Ammoniumsulfatlösung bei 4 Grad C gemischt. Hiebei tritt eine Ausfällung der für die Herstellung der Konjugate erforderlichen Gammaglobuline ein. Die Gammaglobuline werden nun bei 4 Grad $C$ bei etwa 3 000-4, 000 Umdrehungen abzentrifugiert. Der Úberstand (Albumine und andere Serumanteile) wird hierauf verworfen und das Sediment in einem 0,01 mol Phosphatpuffer von $\mathrm{pH} 7,2$ wieder gelöst. Anschließend wird diese gewonnene Gammaglobulinlösung über eine Sephadexsäule (Sephadex G 25 Medium äquilibriert mit 0,15 mol $\mathrm{PO}_{4} \mathrm{NaCl}-\mathrm{Puffer}$ 
pH 7,2) vom restlichen Ammoniumsulfat abgetrennt. Das gereinigte Gammaglobulin wird nun auf seinen Proteingehalt überprüft und mit einer n/10 $\mathrm{NaOH}$ Lösung auf ein $\mathrm{pH}$ von 9-9,5 eingestellt.

Anschließend wird der Globulinlösung je nach Gehalt pro g Protein 5 mg FITC (Serva Heidelberg) beigegeben. Das Ganze wird hierauf 12 Stunden bei 4, Grad C verrührt. Das hiedurch gewonnene Konjugat läßt man hierauf durch eine Säule laufen, die mit DEAE-Zellulose (äquilibriert mit $0,01 \mathrm{~mol}$ $\mathrm{PO}_{4}$ Puffer 7,2) gepackt wurde. Bei diesem Vorgang wird das Konjugat vom überschüssigen Farbstoff befreit. Die Eluierung erfolgte mit verschieden molaren NaCl-Lösungen in 0,01 mol $\mathrm{PO}_{4}$ Puffer pH 7,2 (0,05 mol, 0,1 mol und $0,15 \mathrm{~mol} \mathrm{NaCl}$ ).

Die aus der ersten Kaninchengruppe gewonnenen Konjugate konnten ohne weitere Behandlung sofort verwendet werden. Die Konjugate, gewonnen aus der zweiten Kaninchengruppe, wurden mit einem Pulver getrockneter Bienenlarven abgesättigt, um unspezifische Reaktionen gegen Bienenlarvenantigen zu beseitigen. Die Bienenlarven wurden vorher mit Aceton getrocknet und anschließend gemahlen. Das Bienenlarvenpulver kam in einer Menge von $100 \mathrm{mg} / \mathrm{ml}$ Serum zur Absättigung des Konjugates zur Anwendung. Vor dem Beifügen zum Serum hatte es sich bewährt, das Pulver mit einem 0,15 mol $\mathrm{PO}_{4} \mathrm{NaCl-Puffer}$ pH 7,2 stark anzufeuchten, um nicht zu große Konjugatverluste in Kauf nehmen zu müssen. Bei Zimmertemperatur erfolgte der Absättigungsvorgang eine Stunde lang unter Schütteln. Anschließend wurde die Trennung des Bienenlarvenmateriales vom Konjugat durch Zentrifugieren vorgenommen.

Herstellung der Präparate : Nativausstriche oder Ausstriche von Zentrifugensedimenten auf Objektträgern werden im nativen Zustand oder mit Aceton fixiert und mit dem Konjugat überschichtet. Anschließend bebrütet man den überschichteten Ausstrich in einer feuchten Kammer bei 37 Grad C etwa 30 Minuten. Nach mehrmaligem vorsichtigem Waschen (Gefahr des Abschwemmens des Ausstriches!) mit Pufferlösung (0,15 mol $\mathrm{PO}_{4}$ NaCl-Puffer pH 7,2 und Aqua dest.) wird eine alkalische Glycerinlösung auf den getrockneten Ausstrich aufgebracht und mit einem Deckglas abgedeckt. Das Präparat wird nun im Fluoreszenzmikroskop im UV-Dunkelfeld beurteilt. Weitere Angaben hinsichtlich Immunofuoreszenz bei Wagner (1967).

Ergebnisse : Auf Grund der Versuche mit den beiden verschiedenen Konjugaten wurde festgestellt, daß die Konjugate, die aus Immunsera gegen die unversporten Kulturbakterien hergestellt wurden, keine Reaktion mit Faulbrutsporen ergaben. Die Konjugate färbten aber sehr gut die vegetativen Stäbchen und die Geißelzöpfe an. Die Konjugate gegen die Sporen waren spezifisch für Sporen. Sie färbten die Geißelzöpfe an, aber nicht die vegetativen Formen des B. larvae. Aus diesem Ergebnis ist abzuleiten, daß es mindestens 
zwei gänzlich verschiedene Antigene bei $B$. larvae gibt. Ein Antigen (vermutlich ein somatisches) ist charakteristisch für die vegetativen Stäbchen, während ein weiteres Antigen hochspezifisch für Sporen ist. Mit größter Wahrscheinlichkeit stellt das Geißelantigen, gegen das mit beiden Konjugaten Antikörper nachweisbar waren, ein weiteres selbstständiges und spezifisches Antigen dar. Dies kann aber nicht mit Sicherheit aus diesen Versuchen abgeleitet werden, da beide zur Immunisierung verwendeten Antigene Geißelantigene enthielten.

Ich führte weiters Versuche durch, um die Spezifität der Konjugate zu beweisen. Unter anderem wurden zahlreiche Bakterienstämme, insbesondere Sporenbildner, auf Kreuzreaktionen geprüft : B. cereus (3 Stämme), B. macerans (1 Stamm), B. mesentericus (4 Stämme), B. subtilis (2 Stämme), Sporenbildner aus dem Bereiche der Biene (10 Stämme), B. anthracis (1 Stamm) und diverse Clostridien (15 Stämme).

Unter all diesen Bakterien konnte eine erhebliche Mitreaktion nur bei Milzbrandbakterien festgestellt werden. Diese Kreuzreaktion war aber nicht so ausgeprägt, daß sie zu Verwechslungen führen konnte. Interessant war eine Mit fluoreszenz bei einigen Blastomyzetenarten. Die Hefezellen sind aber morphologisch relativ leicht von einem gut eingelesenen Untersucher abzugrenzen.

Im Verlaufe meiner noch nicht abgeschlossenen Untersuchung an Honigsedimenten und Bienenwintergemüll konnte ich feststellen, daß Faulbrutsporen im Bereiche der Bienenhaltung weiter verbreitet sind, als wir gemeinhin annehmen konnten. Möglicherweise muß die ganze Epidemiologie der Faulbrut neu überdacht werden. Regelmäßig konnte ich in Importhonigen aus Übersee, aber auch aus vielen, vor allem ost-europäischen Ländern, Faulbrutsporen nachweisen. Durch diese neue Untersuchungsmethode ergibt sich in Hinkunft auch eine Kontrollmöglichkeit für Importhonige, die es ermöglichen könnte, stark mit Faulbrutsporen verseuchte Honige auszuscheiden oder zurückzuweisen.

Eingegangen im Juni 1973

Reçu pour publication en juin 1973

\section{RÉSUMÉ}

L'auteur expose les différentes méthodes de laboratoire qui sont utilisées couramment pour le diagnostic de la loque américaine. Alors qu'en règle générale l'apiculteur expérimenté n'a pas besoin d'un examen de laboratoire pour reconnaitre la maladie à un stade avancé, il existe toute une série de cas où seul un tel examen peut donner une réponse claire : ce sont par exemple l'identification d'infections débutantes ou larvées, la mise en évidence de formes de multiplication de $B$. larvae, la recherche des spores de loque dans le miel ou dans les déchets d'hivernage, etc... 
Les possibilités de diagnostic utilisées jusqu'ici ont été étendues par l'introduction récente des méthodes d'immunofluorescence et si bien affinées qu'il est même possible de rendre visible la présence d'un nombre réduit de spores dans un sédiment ou un échantillon de façon spécifique. Jusqu'ici la mise en évidence d'un petit nombre de spores n'était possible que par l'emploi des cultures. Malheureusement il existe dans le miel ou dans les autres matériaux à examiner, d'autres bactéries sporulantes banales et qui sont sensiblement plus résistantes à la chaleur, entre autres facteurs, que les spores de la loque. Les colonies de ces bactéries sporulantes banales croissent sur le milieu de culture avec beaucoup plus de force que $B$. larvae et interdisent souvent par leur dominance sur les colonies de $B$. larvae un diagnostic certain.

Avec l'immunofluorescence ces causes d'erreur disparaissent; cependant il est très diffcile de bien faire adhérer les frottis, qu'il faut laver à plusieurs reprises, à la lame porte-objet. De fortes réactions croisées avec des bactéries sporulantes voisines n'ont pas été constatées. On a étudié 36 souches bactériennes. B. anthracis a donné une réaction concurrente. Il reste la possibilité qu'il existe d'autres bactéries sporulantes banales susceptibles de réagir concurremment, mais cela est peu probable. Mais comme il existe des différences antigéniques nettes entre spores et formes végétatives chez $B$. larvae, il est indispensable, selon les cas, d'utiliser des conjugués appropriés.

\section{LITERATUR}

Forster J. W., Hardwick W. A. \& Beverly Guirard, 1950. Antisporulation factors in complex organic media. J. Bact., 59, 463-470.

KLockow G., 1925. Über die Bildung von Immunstoffen des Bacillus larvae (brandenburgensis), des Erregers der bösartigen Faulbrut der Honigbiene. Erlanger Jahrb. Bienenkunde 3, 29-43.

Poltew W. J., 1958. Serologiceskaja diagnostika nekotornich bakteriosov medonosnoj pseli i drugich nasekomich. Materiali li meschdunar. konf. po patologii nasekomich i biologic. methodu borbi s wrediteljami, Praga.

Schulz-Langner Elis., 1956. Ein neues Bild der bösartigen Faulbrut der Honigbiene. Z. Bienenforsch. 3, 149-180.

Toschkov Al., Valerianov Ts., Tomov A., 1970. Die Immunofluoreszenzmethode und die schnelle und spezifische Diagnostik der amerikanischen Faulbrut bei der Bienenbrut. Bull. apicole 13, 13-18.

Toschkov Al., Valerianov Ts., Tomov A., 1969. Die Immuno fluoreszenzmethode, eine schnelle und spezifische Diagnose der amerikanischen Faulbrut. XXII. Int. Bienenzuchtkongreß d. Apimondia S. 593 München.

Wagner M., 1967. Fluoreszierende Antikörper und ihre Anwendung in der Mikrobiologie. Verlag Gustav Fischer Jena.

Werner F., 1935. Ein Beitrag zur Diagnose der bösartigen Faulbrut der Honigbiene. Wien. tierärztl. Mschr. 22, 257-261.

\section{Anschrift des Verfassers :}

Hochschul-Doz. Dr. Elmar Otte, Tierärztliche Hochschule Wien, Institut für Fisch-und Bienenkunde, 1030 Wien, Linke Bahngasse 11 